\title{
Préface
}

L'Autorité de Sûreté Nucléaire (ASN) assure le contrôle des applications médicales des rayonnements ionisants depuis 10 ans. Après avoir mis en place une règlementation entièrement nouvelle dans le domaine de la radioprotection des patients, dès 2007, elle a centré son action sur la sécurité des soins en radiothérapie.

A partir de 2008, l'ASN a étendu son champ d'action à la radiologie interventionnelle et aux différents actes qui, de plus en plus, font appel aux rayonnements ionisants pour guider le geste médical. La maîtrise de l'augmentation des expositions liées aux examens d'imagerie constitue également une priorité depuis 2011.

La physique médicale est au cœur de ces problématiques liées à la maitrise des doses afin d'obtenir le résultat attendu en ne délivrant que la dose nécessaire (principe d'optimisation, analyse bénéfice-risque) pour le diagnostic ou pour les soins.

Les missions des physiciens médicaux, aussi appelés dans la règlementation française "Personnes spécialisées en radiophysique médicale ", couvrent les champs de l’optimisation, de la qualité et de la sécurité ainsi que la gestion des risques liés aux rayonnements ionisants lors des actes les mettant en œuvre.

Les incidents et accidents survenus en radiothérapie depuis 2005 et plus récemment en radiologie interventionnelle ont mis en lumière le rôle majeur de ces spécialistes et la nécessité de leur implication dans l'ensemble des applications médicales des rayonnements ionisants. Initiée dans le domaine de la radiothérapie, la mise en 
œuvre du management de la qualité et de la gestion des risques lors des processus de soins, est indispensable à la sécurisation et à l'amélioration des pratiques et doit s'étendre à l'ensemble des applications diagnostiques et thérapeutiques.Le besoin de mieux définir le cadre d'exercice du physicien médical en l'appuyant sur des recommandations de «bonnes pratiques " figurait parmi les mesures du plan national pour la radiothérapie préparé fin 2007 sous l'impulsion du Ministère de la Santé, sous l'intitulé " établir un guide de bonnes pratiques en physique médicale ».

Le guide des bonnes pratiques de physique médicale, dont le champ couvre la radiothérapie, la médecine nucléaire et la radiologie, est centré sur la pratique professionnelle du physicien médical pour tout ce qui relève de la qualité et de la sécurité dans l'utilisation médicale diagnostique et thérapeutique des rayonnements ionisants. Il aborde les aspects réglementaires et les principes généraux en matière de qualité et de sécurité lors de la mise en service des équipements et lors des mesures et calculs des doses reçues par les patients. Ce document de référence, à destination des professionnels en vue de les aider dans l'exercice de leur profession, est le reflet des règles de "bonne pratique " établies au sein de la profession au niveau national et international. Il pourra également être utilisé par les professionnels avec lesquels les physiciens médicaux travaillent régulièrement et les représentants des institutions de santé à l'échelon local, régional ou national pour leur permettre de mieux appréhender les conditions d'exercice de cette profession.

Il pourra également servir à d'autres organismes impliqués, par exemple, dans la formation, la recherche, la sécurité, l'économie,... pour qu'ils puissent mieux connaître les caractéristiques et les enjeux de la profession de physicien médical.

La mobilisation des professionnels et des institutions demeure un préalable pour parvenir à une réelle application du principe d'optimisation, de maîtrise des doses délivrées aux patients et contribuer à la sécurité des soins. Ce document, issue du consensus des professionnels, est un élément clé dans cette démarche.

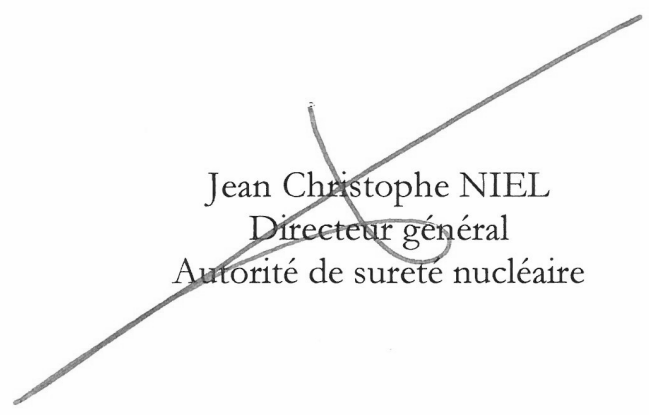




\section{Ont participé à cet ouvrage}

Groupe de pilotage (SFPM) :

Jean-Claude Rosenwald, Chef de projet

Christophe Aventin

Frédéric Coste

Pascal François

Chantal Ginestet

Bénédicte Perrin

Cécile Salvat.

Groupe de cotation :

Olivier Caselles (SFPM)

Véronique Dedieu (SFPM)

Catherine Dejean (SFPM)

Alain Batalla (SFPM)

Bonniaud Guillaume (SFPM)

Dominique Le Du (SFPM)

Albert Lisbona (SFPM)

Vincent Marchesi (SFPM)

Thierry Sarrazin (SFPM)

Jean-Jacques Mazeron (SFRO)

Francis Lipinski (SFRO)

Pierre Vera (SFMN)

Maximilien Vermandel (SFMN)

Hubert Ducou le Pointe (SFR)

Vincent Vidal (SFR).

Représentants institutionnels :

Cécile Henry, INCa

Marc Valéro, ASN.
Assistance méthodologique :

Valérie Mazeau-Woynar, INCa.

Assistance bibliographie et site internet :

Camille Prot, INCa.

Groupe de lecture ${ }^{1}$ :

Bernard Aubert (IRSN)

Manuel Bardies

Hélène Beauvais

Pierre Bey

André Costa

Claire Desblancs

Cécile Etard (IRSN)

Teresa Eudaldo (Espagne)

Bardia Farman

Régis Ferrand

Robin Garcia

Jean-Yves Giraud

François Husson

Christine Jimonet (INSTN)

Malick Koulibaly

Loïc de Carlan

Jean-Pierre Manens

Suzanne Naudy

Alain Noel

Pierre Pilette (Belgique)

Amélie Roué (INSTN)

Julie Sage (AFSSAPS)

Francis Verdun (Suisse).

1. Pour préserver l'indépendance des membres du groupe de lecture, ne sont mentionnées que les appartenances à des organismes institutionnels qu'ils représentent ou à des pays européens autres que la France. 


\section{Remerciements}

Nous tenons à remercier, pour leurs remarques et leur contribution à la rédaction de cet ouvrage, les personnes suivantes :

Aurélien Bouëtté, Stephen Breen, André Bridier, Bruno Chauvenet, Jean Chavaudra, Isabelle Gardin et Karine Herlevin 